\title{
Using Flexible Ethernet to Build a Flexible Network
}

\author{
Zijian $\mathrm{He}^{*}$, Xinyuan Wang* \\ * IP Technology Research Dept., Huawei Technology Co., Ltd., Beijing, China. \\ hezijian@huawei.com, Wangxinyuan@huawei.com
}

\begin{abstract}
Based on network processor and traffic management processor, packet devices can generate flows with specified bandwidths. Unfortunately, fixed-bandwidth Ethernet interface hides the flexibility of packet devices and packet devices can just provide single flow with fixed bandwidth. Flexible Ethernet introduced in this paper can divide Ethernet interface into several sub-Ethernet interfaces and the bandwidth of each subEthernet interface is configurable. Based on this feature, flexible Ethernet can be used to build a flexible network for traffic optimization, and it also can be used for IP\&Optical synergy towards IP offloading.
\end{abstract}

Keywords — Flexible Ethernet, Flexible Bandwidth, Flexible Network, IP\&Optical Synergy, IP Offloading, Network Optimization

\section{INTRODUCTION}

Continuing growth of network bandwidth, including both user traffic and machine-to-machine traffic [1], promotes higher bandwidth of IP network and Optical network generation after generation. As the demands of bandwidth for client and core network are diverse and variable in real Ethernet/IP networks, flexibility is becoming more and more important because operational ease and network optimization can benefit from flexibility. For example, in order to optimize traffic in inter-date-center, flexible Ethernet for IP device and flexible optical network are both in demand so that the bandwidth between nodes can be configured by demands [2]

On one hand, for optical network, as the development of ODUflex [3], flexible grid, bandwidth variable transponder (BVT), flexible ROADM and flexible OTN [4], flows with flexible bandwidth can be carried by optical network. It means that optical network is prepared for flexible bandwidth flows. On the other hand, packet devices such as Ethernet Router/Switch can handle flexible bandwidth flows essentially. In packet devices, packets traverse through Network Processor (NP) unit and Traffic Management (TM) unit. The two units have strong ability for flow bandwidth control. Based on the two units, Ethernet Router/Switch can generate different flows with different bandwidth by demands easily. However, for Ethernet Router/Switch, the bandwidths of Ethernet interfaces defined by IEEE 802.3 [5] are fixed rates, such as 10G, 40G, $100 \mathrm{G}$ and $400 \mathrm{Gbps}$ for each generation. Due to fixed-rate Ethernet interface limitation, the flexibility of Ethernet Router/Switch is hided. Figure 1 shows that the fixed-rate Ethernet is the bottleneck of current IP\&optical synergy for a flexible network.

In order to exert the potential of flexibility in packet devices to build a flexible network, flexible Ethernet is in demand. The purpose of this paper is to introduce a method to implement flexible Ethernet that can divide the Ethernet interface into several independent sub-Ethernet interfaces with various bandwidths by configuration.

The rest of the paper is organized as follows. Section II introduces the concept of flexible Ethernet and how flexible Ethernet implemented. Section III describes two application scenes of flexible Ethernet: building a flexible network using flexible Ethernet for network optimization in data center and IP\&Optical network synergy for IP offloading. Section IV makes a conclusion for this paper.

\section{FLEXIBLE ETHERNET}

Ethernet interfaces defined by IEEE802.3 are widely used in various networks. Flexible Ethernet is a supplement of current Ethernet interfaces to meet the requirement for flexibility of interface to build a flexible network. The architecture of flexible Ethernet inherits the architecture of fixed-rate Ethernet and only a few changes are made on PCS layer. After adding some special logic modules on PCS layer, the Ethernet interface can be divided into several independent sub-Ethernet interfaces and each sub-Ethernet interface occupies several physical lanes and provides flexible bandwidth.

\section{A. Formal definition of flexible Ethernet interface}

Assumed that the interface of flexible Ethernet is composed of $N$ physical lanes, the bandwidth of each lane is $B$ and the total bandwidth provided by flexible Ethernet is $B_{T}$. It is

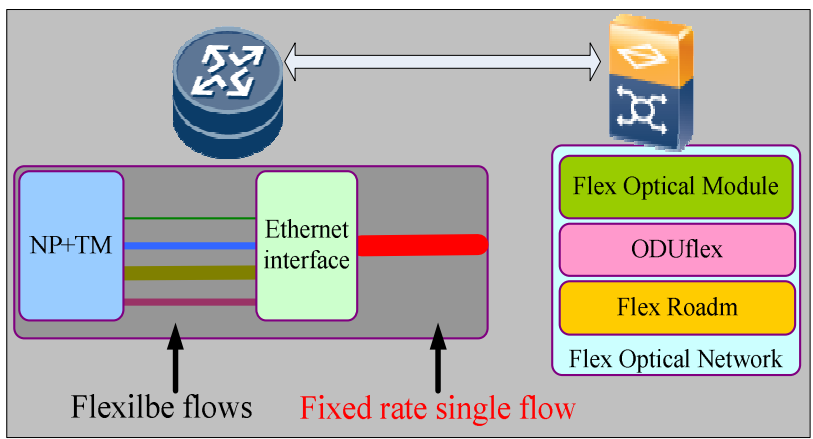

Figure 1. NP\&TM can provides flexible flows and flexible optical network can carries flexible flows. Fixed-rate Ethernet is the bottleneck of current IP\&optical synergy for a flexible network. 
obvious that $B_{T}=N^{*} B$. Flexible Ethernet can provides $N$ subEthernet flows at most and each sub-Ethernet flow is defined by $F_{i}, \mathrm{i} \in[1, \mathrm{~N}]$. The bandwidth of $F_{i}$ is defined by $B_{i}$ and occupies $L_{i}\left(L_{i} \in[0, \mathrm{~N}]\right)$ independent physical lanes, it means that each physical lane belongs to just one flow. The sub Ethernet interfaces provided by flexible Ethernet can be defined as follow:

$$
\begin{aligned}
& B_{T}=B_{1}+B_{2}+\cdots+B_{N} \\
& B_{i}=L_{i} * B \\
& N=L_{1}+L_{2}+\cdots+L_{N}
\end{aligned}
$$

According to formula 1 3, flexible Ethernet can provides various combinations of sub-Ethernet interfaces with granularity of B. Figure 2 shows a $400 \mathrm{G}$ Ethernet interface which is composed of 16 physical lanes and the bandwidth of each lane is $25 \mathrm{G}$. Using flexible Ethernet, the interface is divided into 5 sub-Ethernet interfaces, the bandwidth of each interface is $25 \mathrm{G}, 100 \mathrm{G}, 175 \mathrm{G}, 50 \mathrm{G}$ and $50 \mathrm{G}$ and each interface occupies one, four, seven, two and two physical lanes.

\section{B. Additional logic modules}

Based on fixed-rate Ethernet, some special logic modules are designed to support the feature of flexibility. For egress side, three modules including stream distributor, flexible container and lane distributor are added in flexible Ethernet. For ingress side, three modules including stream aggregator, flexible container and lane aggregator are added in flexible Ethernet.

The input of flow distributor is a full bandwidth stream and the outputs of flow distributor are $N$ independent sub-flows sending to flexible containers. Flow distributor recognizes flows from input stream and dispatch each of them to one of $N$ output sub flows. The dispatch algorithm can be defined based on some sections of packets from input stream. The functionality of flow aggregator is opposite from that of flow distributor. The inputs of flow aggregator are $N$ independent sub-flows from flexible containers and the output of flow aggregator is a full bandwidth stream. Flow aggregator aggregates all input flows into one flow.

Flexible container plays an important role in flexible Ethernet implementation. The input of flexible container is a sub flow from flow distributor or a sub flow from lane

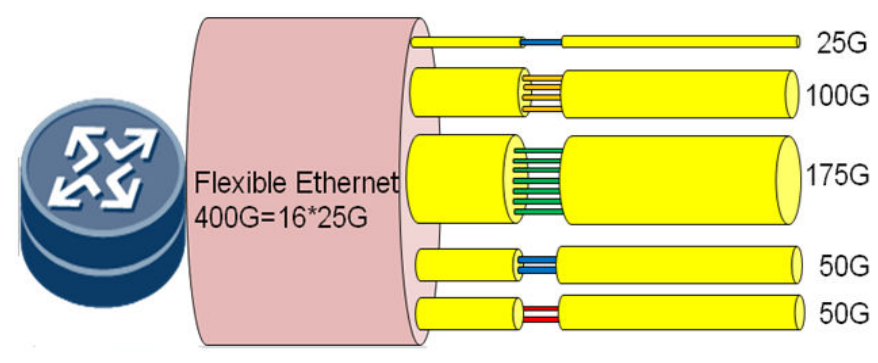

Figure 2. A $400 \mathrm{G}$ Ethernet interface which is composed of 16 lanes and the bandwidth of each lane is $25 \mathrm{G}$. Flexible Ethernet divide the interface into 5 sub-Ethernet flows: $25 \mathrm{G}, 100 \mathrm{G}, 175 \mathrm{G}, 50 \mathrm{G}$ and $50 \mathrm{G}$ aggregator. The output of flexible container is a fixed-rate flow to lane distributor or flow aggregator. Corresponding to flexible flows $F_{1}$ to $F_{N}, N$ flexible containers are built to serve each flexible flow. Flexible container can be read and write with various bandwidths by configuration and the maximum bandwidth supported by flexible container supported is $B_{T}$.

The input of lane distributor is a sub flow from flexible container. According to configuration, several specified physical lanes are used to load the sub flow. Lane distribute responsible for loading one sub flow onto specified physical lanes. The functionality of lane aggregator is opposite from that of lane distributor. It receives streams from specified lanes, recover packets and then send to flexible container.

\section{Flexible Ethernet implementation}

The following workflow omits the procedures of encode/decode, scramble/descramble etc. that almost the same with fixed-rate Ethernet.

For Egress side, full bandwidth stream are send to flow distributor first, and then the flows in streams are recognized and dispatched to specified flexible container. Sub flows in flexible containers are shaped and send to lane distributor with fixed rate. Lane distributor then loads the fixed-rate flow to specified physical lanes by configuration. The full workflow is shown as Figure 3.

For ingress side, data streams from lanes are grouped and aggregated in lane aggregator. Packets are recovered and write to flexible container. After that, packets are read out from all flexible containers by flow aggregator and one full bandwidth

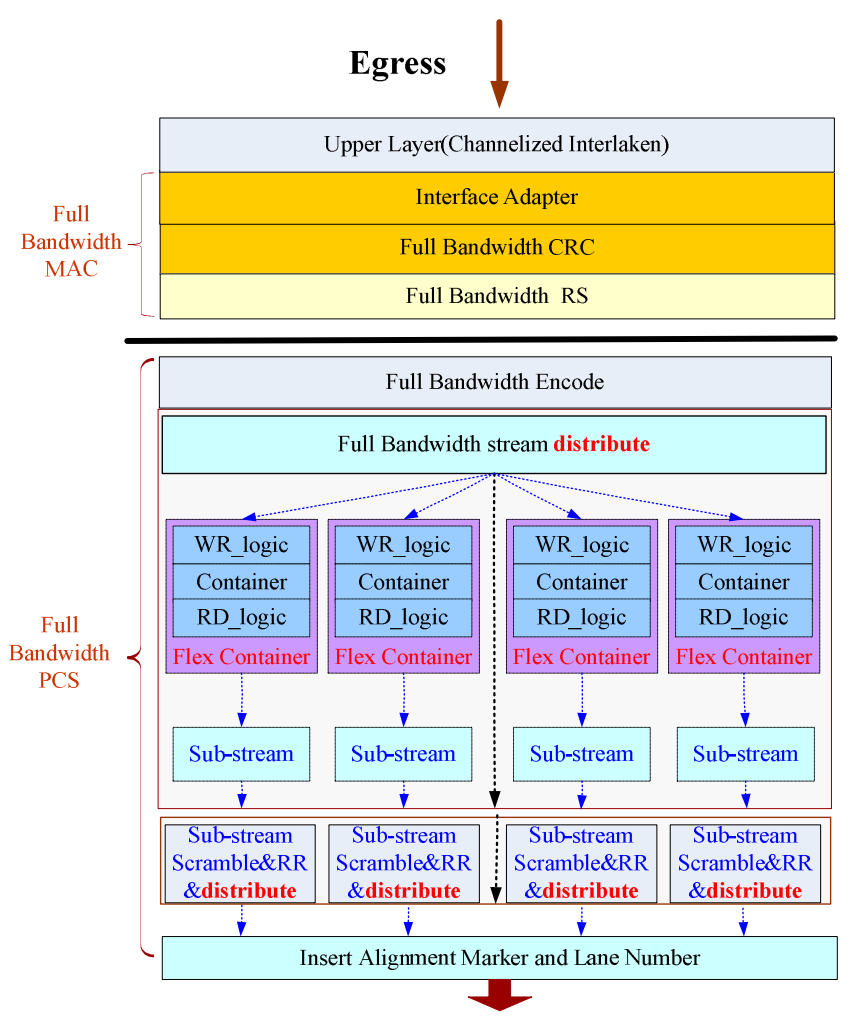

Figure 3. Workflow of egress side for fleixble Ethernet 
stream is merged. The full workflow is shown as following Figure 4.

\section{Cost of flexible Ethernet}

One method to achieve flexible Ethernet is to combine several copies of Ethernet interface logic design and each with different bandwidth allocating configuration. This method is logic resource waste and only limited number of bandwidth configurations can be supported because of logic resource limitation. In flexible Ethernet, the bandwidth of sub-Ethernet interface is configurable, that means flexible Ethernet can provides various combination of bandwidths just by configuration.

The additional cost of the flexible Ethernet is limited. Although more logic resources are consumed compared to fixed-rate Ethernet, it is much worthy to adopt flexible Ethernet as it can support various bandwidth combination instead of changing interface card. About 35\% logic resources more than fixed-rate Ethernet is needed according to our experiment for $400 \mathrm{G}$ flexible Ethernet.

\section{III.APPLICATION OF FLEXIBLE ETHERNET}

By using flexible Ethernet, the Ethernet interface can break up into several sub-Ethernet interfaces with any bandwidth by demands and each sub-Ethernet interface is physically independent by occupying several physical lanes exclusively. There are many benefits can be achieved based on the flexibility of flexible Ethernet. We list two application scenarios as follow.

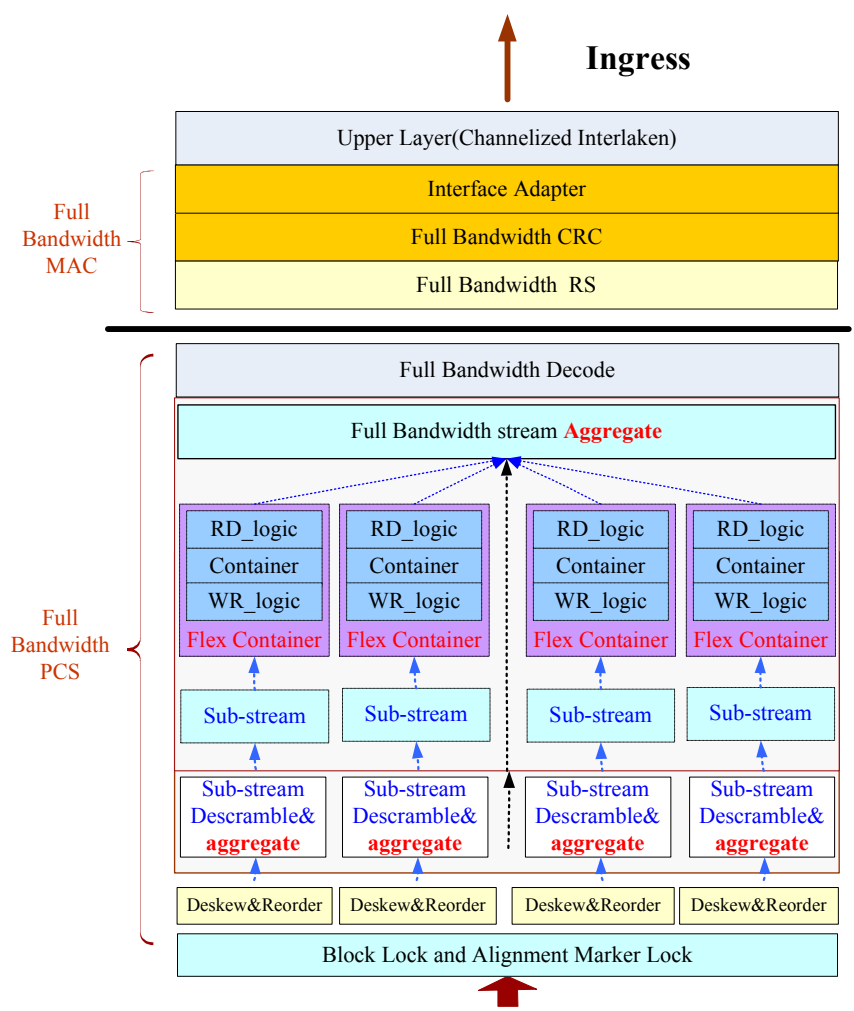

Figure 4. Workflow of ingress side for flexible Ethernet

\section{A. Flexible Ethernet for IP\&Optical synergy}

Our assumptions about IP\&Optical synergy can be concentrated to Figure 5. At level 3, all application data flows are all carried by IP layer. At level 2, flows from IP layer are processed and flow ids are generated by configuration. At level 1, flexible PCS is used to aggregate flows with same flow id and each aggregated flow is distributed to sub Ethernet channel according to configuration. At level 0 , flexible flows are carried by flexible optical network with $\lambda$ switch.

Packet devices can provide flexible bandwidth flows by using flexible Ethernet. At the same time, optical networks can carry flexible bandwidth flows by using ODUflex, flexible Grid, BVT, flexible ROADM and flexible OTN. A flexible and configurable network can be built by IP\&optical network synergy. Figure 6 illustrates IP device connected to Optical device through Flexible Ethernet. First, flows with specified bandwidths and flow ids are sent to flexible Ethernet after NP\&TM processing. According to configuration, these flows are distributed to several flexible containers. For each flexible container, there is a corresponding sub-Ethernet channel connects to OTN. For each channel, data flow received in OTN is mapped to ODUflex using GFP-F, and then ODUflex can be multiplexed to high order ODU or transmitted by flexible ROADM directly.

There are many benefits to use flexible Ethernet for IP\&optical synergy. As all sub-Ethernet interfaces provided by flexible Ethernet are independent, optical network devices with flexible Ethernet can recognize each flow easily when the configuration of flexible Ethernet is shared by optical network device. This can be achieved by management channel between IP network and Optical network. Besides that, the bandwidth of each flow is transparent to OTN through management channel. Without flexible Ethernet, VLAN is recognized and used to distinguish different flows in optical network device. With flexible Ethernet, IP offloading can be applied more easily and flexibly compared to VLAN. As each sub-Ethernet flows are physical independent, optical network device can recognize flows naturally. The bandwidths of each subEthernet flow are configurable, optical network device can adapt to flexible bandwidth just by getting the configuration without detecting the input rates of flows.

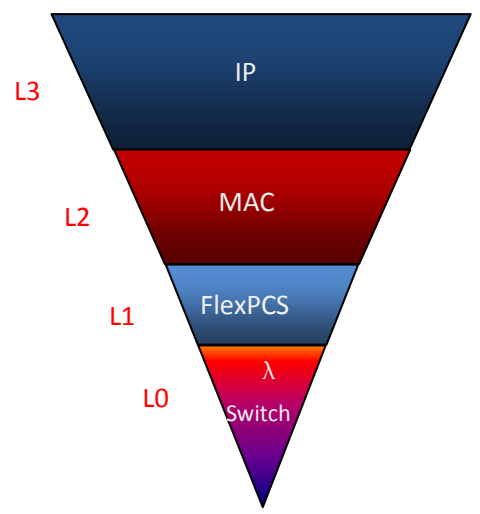

Figure 5. The concept of IP\&Optical synergy using flexible Ethernet 


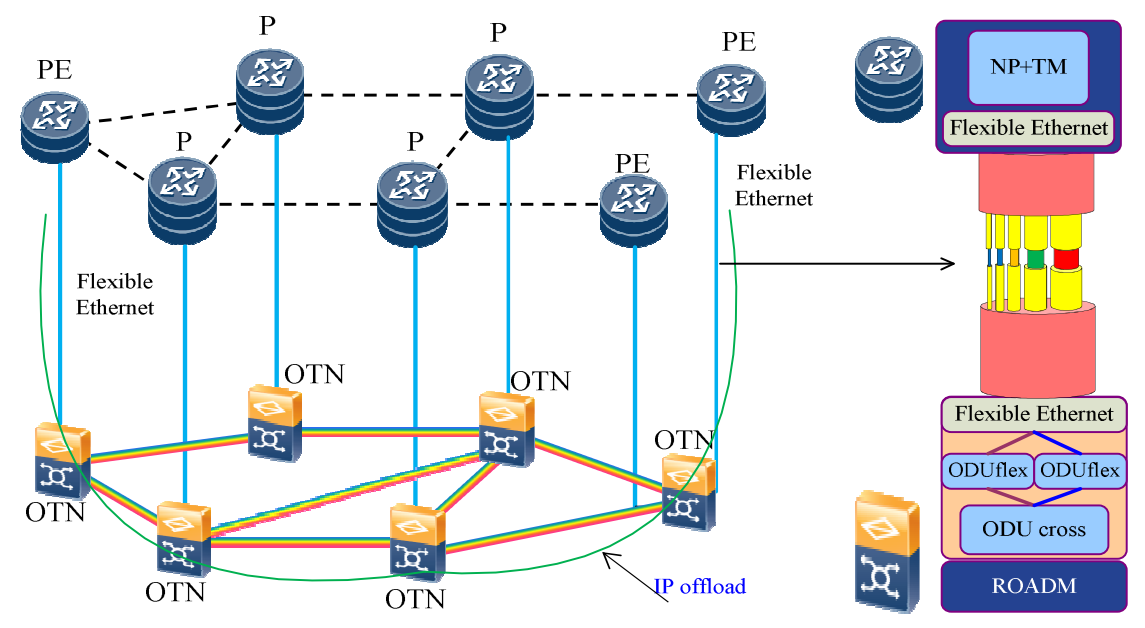

Figure 6. IP\&Optical synergy for IP offloading.

\section{B. Flexible Ethernet for network optimization in data center}

As the east-west flow is becoming the main data flow in data center, the network cost is getting much higher for data center. It is very attractive for network optimization in data center.

Generally speaking, the bandwidth demands between different nodes (refers to Router/Switch/Storage/Server etc.) are different according to operations and algorithms processed in data center. Fixed-rate Ethernet interface can only connect to one other node while flexible Ethernet interface enables nodes connect to several other nodes with specified bandwidth by demands.

Using flexible Ethernet, the bandwidth of one port can be divided to multi-sub ports with different bandwidths by configuration. The configuration can be generated from the algorithms processed in data center. As one port can connect to several other nodes and the bandwidth of the port is shared by these sub ports, the bandwidths of these ports can be variable without wasting the bandwidth of port bandwidth or reserve extra bandwidth, so that the bandwidth of nodes can be fully used according to requirement. An example of flexible network build by flexible Ethernet can be shown as Figure 7. Based on this feature, the network of data center can be optimized by adjusting the bandwidth between nodes.

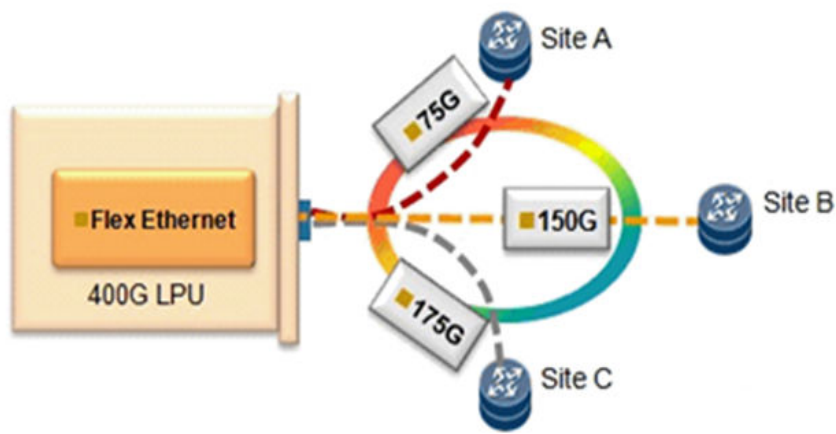

Figure 7. One $400 \mathrm{G}$ port is divided into three sub interfaces by demand and connect to three different packet devices.

\section{IV.CONCLUSIONS}

With limited additional logic resource compared to fixedrate Ethernet, flexible Ethernet can divide the interface into several sub-Ethernet interfaces with various bandwidth combinations by configuration. It can be used to build a flexible network for IP\&Optical synergy and traffic optimization in data center.

\section{REFERENCES}

[1] Vijay Vusirikala, Cedric Lam, Paul Schultz and Bikash Koley, "Drivers and Applications of Optical Technologies for Internet Data Center Networks," Proc. OSA/OFC/NFOEC, 2011.

[2] Xiaoxue Zhao, Vijay Vusirikala, Bikash Koley, Valey Kamalov, and Tad Hofmeister, "The Prospect of Inter-Data-Center Optical Networks," IEEE Communications Magazine, vol.51 Issue 9, pp32-38, September 2013.

[3] "Hitless adjustment of ODUflex(GFP)," http://www.itu.int/rec/T-RECG.7044/en

[4] Zong Liangjia, Liu Ning, and Ma Teng, "FBON makes your fiber flexible,'http://www.huawei.com/en/abouthuawei/publications/communicate/hw-145798.htlm

$\begin{array}{llll}\text { [5] IEEE } & \text { standard } & \text { for } & \text { Ethernet. }\end{array}$

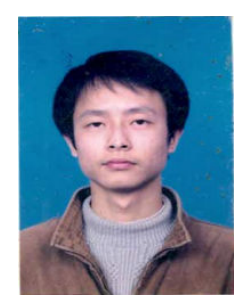

Zijian He received the B.S. degree from Wuhan University of Computer Science and Technology, China, and the Ph.D. degrees from the Institute of Computing Technologies, Chinese Academy of Sciences in 2005 and 2011, respectively. $\mathrm{He}$ is currently a research engineer in fixed network research department in Huawei Technologies Co. Ltd., Being, China. He is responsible for key technology research for IP router

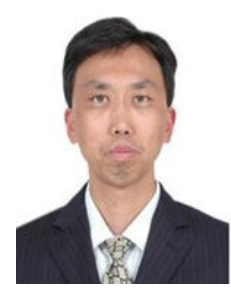

Xinyuan Wang received the M.S. degree in electrical engineering from Hebei University of technology, China. He is a chief IP technology researcher at fixed network research department in Huawei Technologies Co. Ltd., Beijing, China. He is responsible for key technology research for IP router, the definition and development of high speed Ethernet technology, and is active in 802.3 400GE study group. 